\title{
Disfunção Endotelial no Diabetes Melito Tipo 1
}

revisão

\author{
Marcello Casaccia Bertoluci \\ Gislaine Vissoky Cé \\ antônio Marcos V. da Silva \\ Marcia K. C. Puñales
}

Departamento de Medicina Interna da Universidade Federal do Rio Grande do Sul (UFRGS),

Porto Alegre, RS, Brasil (MCB);

Programa de Pós-Graduação em Ciências Médicas da UFRGS

(MCB, GVC); Programa de

Pós-Graduação em Clências Biológicas: Fisiologia da UFRGS

(AMVS); Universidade de Santa

Cruz do Sul (Unisc) (AMVS);

Instituto da Criança com

Diabetes (ICD) do Grupo

Hospitalar Conceição, Porto

Alegre, RS, Brasil (GVC, MKCP).

As complicações vasculares são a maior causa de morbimortalidade em pacientes com diabetes. Os mecanismos envolvidos no desenvolvimento das doenças micro e macrovasculares são complexos e parcialmente compreendidos, mas se iniciam invariavelmente por um endotélio que se torna disfuncionado. O óxido nítrico é um importante regulador da função endotelial e o comprometimento da sua atividade é fator determinante para a disfunção endotelial (DE). No diabetes tipo 1, diversos fatores, como a hiperglicemia aguda, mau controle glicêmico crônico, tempo de diagnóstico e presença de neuropatia autonômica ou microalbuminúria estão associados à DE. Tanto o estresse oxidativo, como a ativação da via dos polóis, via da proteína quinase C e formação dos produtos avançados de glicação não-enzimática são potenciais mecanismos patogenéticos da DE. A detecção precoce da disfunção endotelial tem valor prognóstico para o desenvolvimento de complicações vasculares e pode ser importante em estratégias de prevenção primária de eventos cardiovasculares no diabetes tipo 1. (Arq Bras Endocrinol Metab 2008;52/2:416-427)

Descritores: Disfunção endotelial; Diabetes tipo 1; Óxido nítrico; Aterosclerose

\section{ABSTRACT}

\section{Endothelial Dysfunction in Type 1 Diabetes.}

Vascular complications are the main cause of mortality and morbidity in diabetes. Mechanisms involved in the development of micro and macrovascular disease are complex and partially understood, but invariably begin as a dysfunctional endothelium. Nitric oxide is an important regulator of endothelial function and the impairment of its activity is determinant of the endothelial dysfunction. In type 1 diabetes, many factors like acute, chronic and postprandial hyperglycemia, as well as the duration of diabetes or autonomic neuropathy and microalbuminuria are associated to endothelial dysfunction. Oxidative stress, polyol pathway activation, protein kinase $\mathrm{C}$ activation and the presence of advanced glycation end-products are potential mechanisms involved in the development of endothelial dysfunction. Early detection of endothelial dysfunction has prognostic value for the development of vascular complications and may be important in strategies for primary prevention of cardiovascular endpoints in type 1 diabetes. (Arq Bras Endocrinol Metab 2008;52/2:416-427)

Keywords: Endothelial dysfunction; Type 1 diabetes; Nitric oxide; Atherosclerosis

\section{INTRODUÇÃo}

Recebido em 11/12/2007 Aceito em 16/12/2007

S DOENÇAS MICRO e macrovasculares são a principal causa de morbimortalidade em pacientes com diabetes melito (1-3) No diabetes melito tipo 
l (DMl), o risco de morte por doença cardiovascular antes dos 60 anos é três a seis vezes maior quando comparado a indivíduos sem diabetes. No entanto, os fatores de risco tradicionais para doença coronária não explicam a totalidade deste excesso de risco $(4,5)$.

A relação entre DMl e doença cardiovascular é bem conhecida (6) e tem sido atribuída à associação entre hiperglicemia crônica, disfunção endotelial (DE) e inflamação crônica $(7,8)$. É bem conhecido, em estudos clássicos, que a hiperglicemia crônica é um importante preditor de complicações micro e macrovasculares $(1,2,9)$.

A DE tem sido sugerida como um evento precoce na patogênese das complicações vasculares do DMl (10). Ela reflete a presença de um fenótipo propenso a aterogênese e pode, dessa forma, servir de marcador de risco para aterosclerose, já tendo sido demonstrada constituir-se em um fator de risco independente para eventos cardiovasculares (11). Nesta revisão abordamos as evidências clínicas, os potenciais mecanismos patogenéticos e os métodos diagnósticos disponíveis para detecção da DE associada ao DMl na prática clínica.

\section{ENDOTÉLIO NORMAL E DE}

O endotélio vascular forma a camada celular que está em contato direto com o lúmen vascular e é separado da camada muscular lisa pela membrana basal. Seu papel é a manutenção da homeostase da vasculatura por meio da síntese de substâncias vasoativas que modulam o tônus vascular, inibem a agregação plaquetária e a proliferação das células musculares lisas vasculares. $\mathrm{O}$ óxido nítrico (NO) tem papel fundamental neste equilíbrio (12).

$\mathrm{Na}$ célula endotelial, o NO é sintetizado a partir da L-arginina pelo óxido nítrico sintase endotelial (eNOS), na presença de oxigênio, NAPH e $\mathrm{BH} 4$ (tetrahidrobiopterina). Rapidamente, o NO se difunde do endotélio para a camada de células musculares lisas e plaquetas, onde ativa a guanilato ciclase ( $\mathrm{Gca}$ ) com conseqüente produção de GMP cíclico (GMPc). A presença do GMPc promove relaxamento vascular e inibição da agregação plaquetária. A meia-vida do NO é de apenas alguns segundos, sendo rapidamente oxidado a nitrato antes de ser excretado na urina (13).

A ativação da eNOS é determinada por fatores como o estresse de cisalhamento (shear stress) causado pelo fluxo sanguíneo na parede luminal, pelo estiramento da parede vascular e pela baixa tensão de oxigê- nio na parede vascular $(14,15)$, cujo sinal é modulado pela acetilcolina (Ach), bradicinina e inibidores da cálcio-ATPase. Por outro lado, a secreção basal de NO é inibida pela $\mathrm{N}^{\mathrm{G}}$-monometil-L-arginina (L-NMMA), um inibidor específico de eNOS $(16,17)$.

Em situações patológicas, como o diabetes melito, pode ocorrer o desacoplamento do eNOS, uma situação em que a transferência de elétrons na cadeia oxidativa não se completa adequadamente. Os elétrons vazam e são captados pelo oxigênio molecular, gerando radicais livres, como o superóxido. O desacoplamento do eNOS é, portanto, um importante mecanismo inicial de DE (18).

A DE é caracterizada pela perda das propriedades do endotélio, isto é, alteração na síntese de proteínas, aumento do tônus vascular, aumento da permeabilidade vascular e aquisição de atividade pró-trombótica e antifibrinolítica. Os principais determinantes são: a diminuição da disponibilidade de $\mathrm{NO}$ e a preponderância de fatores vasoconstritores liberados pelo endotélio, em detrimento aos fatores vasodilatadores (19). A DE gera, portanto, alteração no perfil antiaterogênico, promovendo migração e proliferação de células musculares lisas, agregação plaquetária, oxidação do LDL, adesão de monócitos e plaquetas e síntese de citocinas inflamatórias, contribuindo para a aterogênese (20).

\section{MÉTODOS DE AVALIAÇÃO DA FUNÇÃO ENDOTELIAL}

A função endotelial pode ser investigada de maneira invasiva ou não-invasiva, com várias técnicas, em diferentes leitos vasculares e por diversos estímulos farmacológicos ou mecânicos. As artérias coronárias podem ser avaliadas em resposta à infusão de acetilcolina, da bradicinina ou pela indução de estresse de cisalhamento (shear stress), com a utilização de angiografia biplanar quantitativa para mensuração das mudanças do diâmetro vascular (21). Outra técnica invasiva é a pletismografia de oclusão venosa para mensuração do fluxo sanguíneo do antebraço em resposta à infusão de acetilcolina na artéria braquial (22). A natureza invasiva destas técnicas, no entanto, envolvendo a canulação de uma artéria e a infusão de drogas vasoativas (23), torna inviável a sua ampla utilização na prática clínica. Assim, as técnicas que envolvem procedimentos não-invasivos ou com infusões de drogas a baixas concentrações e com efeitos somente em pequenos segmentos vêm sendo cada vez mais utilizadas. 


\section{Técnicas não-invasivas (Tabela 1)}

A avaliação da função endotelial de maneira não-invasiva pode ser feita pela captação de imagens da artéria braquial por ultra-sonografia de alta resolução (23). A técnica mais comumente utilizada envolve a hiperemia reativa e o estresse de cisalhamento, os quais são os estímulos para a ocorrência de dilatação mediada pelo fluxo sanguíneo (DMF), ou seja, a vasodilatação dependente do endotélio (23). A hiperemia reativa ocorre após um período de isquemia induzido por oclusão da artéria braquial com um manguito aneróide insuflado, ocorrendo liberação progressiva de mediadores vasodilatadores, como a adenosina e íons $\mathrm{H}+$ pelo tecido isquêmico. Ao ocorrer a liberação do fluxo arterial, surge a hiperemia reativa, com o fluxo sanguíneo aumentando de $100 \%$ a $300 \%$ em relação ao basal, promovendo estresse de cisalhamento e permitindo a entrada de cálcio intracelular que ativa o eNOS, liberando NO (24). Este mecanismo depende da presença do $\mathrm{NO}$ e de integridade funcional do endotélio.

O procedimento técnico da DMF consiste na medida do diâmetro longitudinal da artéria braquial em condições basais seguido pela oclusão da artéria durante 5 minutos, com medida do diâmetro arterial após 45 e 60 segundos da liberação da oclusão (25). O dado obtido é o percentual de dilatação após a hiperemia reativa em relação ao basal. Considera-se DE quando o aumento da dilatação for inferior a $10 \%$.

Para isolar a presença de disfunção de músculo liso vascular, é necessário avaliar, também, em procedimento subseqüente pela mesma técnica, a vasodilatação independente do endotélio. Após 10 minutos de repouso, para o restabelecimento das condições iniciais pós-hiperemia reativa, realizam-se novas medidas basais e administra-se nitroglicerina sublingual $(0,4 \mathrm{mg})$. Após 4 minutos, mede-se o diâmetro da artéria. Esta diferença representa a vasodilatação independente do endotélio, ou seja, mediada pelo músculo liso vascular. Considera-se normal uma dilatação acima de $10 \%$ em relação ao basal (26).

Outra técnica não-invasiva que vem sendo utilizada é a tonometria de artéria periférica pela hiperemia reativa $(27,28)$, que avalia a função endotelial pela combinação da dilatação mediada por fluxo e pelas medidas da amplitude de onda de pulso arterial por meio de um sensor pneumático posicionado no dedo indicador. A reatividade microvascular da pele do antebraço ou do dorso do pé (23) é avaliada por fluxometria com laser Doppler, tendo como estímulo vasodilatador, dependente do endotélio, a iontoforese de acetilcolina.

A técnica de complacência da veia dorsal da mão é um método minimamente invasivo, descrito por Aellig em 1981 (29) e recentemente utilizada por nosso grupo (30). Consiste na infusão de drogas vasoativas em uma veia superficial do dorso da mão, somente com efeitos locais, para mensurar a vasodilatação dependente do endotélio em resposta a acetilcolina, bradicinina ou isoprenalina.

Outra técnica que vem sendo cada vez mais utilizada é a medida da espessura da camada íntima-média da carótida comum (IMC) por ultra-sonografia, a qual representa um marcador estrutural de aterosclerose e correlaciona-se inversamente com a DMF na artéria braquial (11).

A pletismografia de oclusão venosa pode ser utilizada para medir as alterações no fluxo sanguíneo do antebraço em resposta à hiperemia reativa $(31)$ e, dessa forma, caracteriza-se também como um método não-invasivo para avaliação da função endotelial.

\section{ALTERAÇÕES MORFOLÓGICAS DO ENDOTÉLIO NO DIABETES}

Alterações na morfologia vascular no diabetes têm sido relatadas tanto em estudos experimentais como em es-

Tabela 1. Métodos não-invasivos de avaliação da função endotelial.

\begin{tabular}{lll}
\hline Técnica & Leito Vascular & Estímulo \\
Ultra-sonografia de alta-resolução (25) & Artéria braquial & Hiperemia reativa \\
Tonometria de artéria periférica $(27,28)$ & Artérias do membro superior & Hiperemia reativa \\
Fluxometria com laser Doppler (23) & Microcirculação da pele & lontoforese de ACh \\
Dorsal Hand Vein (29,30) & Veia do dorso da mão & ACh, bradicinina \\
Pletismografia de oclusão venosa (31) & Veias do antebraço & Hiperemia reativa \\
\hline
\end{tabular}


tudos clínicos. Em aorta de coelhos com diabetes induzido por aloxana, alterações endoteliais são visíveis duas semanas após o início da hiperglicemia e tornam-se mais graves após seis semanas de diabetes (32). Estas incluem a adesão de leucócitos, plaquetas e material fibrinoso na superfície endotelial. Em ratos, após seis semanas do início do diabetes induzido pela estreptozotocina (STZ), também se observa alterações, como o aumento da permeabilidade endotelial e morte celular endotelial (33).

Em estudos clínicos clássicos, o achado encontrado mais importante é o espessamento da membrana basal, observado em capilares da pele de pacientes com DMl comparado a indivíduos não-diabéticos (34). Esta alteração parece ser reversível, uma vez que o controle glicêmico intensivo durante um ano é capaz de reduzir a espessura da membrana basal em capilares musculares de pacientes com DMl (35). Estudos em células endoteliais obtidas de cordão umbilical de gestantes com DMl comparadas a gestantes sem diabetes, mostraram aumento da área mitocondrial (36). O significado clínico destas alterações não é claro, porém podem contribuir para a instalação e progressão da doença aterosclerótica.

\section{ALTERAÇÕES FUNCIONAIS NO ENDOTÉLIO NO DM1}

Existem diversas evidências da ocorrência de DE no diabetes melito. Vários investigadores demonstraram disfunção vasomotora em modelos experimentais $(37,38)$ e em estudos clínicos, tanto em DMl $(39,40)$ como em DM2 (41,42).

A função endotelial no diabetes tipo 1 é modulada pelo grau de hiperglicemia (43), pela duração do diabetes $(10,44,45)$, pelas concentrações séricas de insulina (46) e pela presença de complicações crônicas, especialmente neuropatia autonômica (47) e microalbuminúria $(48)$.

\section{RESPOSTA VASCULAR À HIPERGLICEMIA AGUDA EM INDIVÍDUOS NORMAIS}

Em indivíduos normais expostos agudamente a altas concentrações de glicose por infusão de dextrose $50 \%$ durante 6 horas, há atenuação da resposta vasodilatadora arterial endotélio-dependente induzida por metacolina, indicando a presença de DE aguda (43). A resposta vasodilatadora ao nitroprussiato, que mede dilatação não- mediada pelo endotélio, parece estar preservada, indicando que o aumento agudo da glicemia pode causar $\mathrm{DE}$, porém este não seria suficiente para causar disfunção de músculo liso vascular. $\mathrm{O}$ efeito observado parece ser específico da glicose, pois a infusão de agente vasodilatador, como o verapamil, e substâncias osmóticas, como o manitol, não alteraram a resposta vasodilatadora do NO induzido pela metacolina (43).

Nem todos os estudos, entretanto, demonstraram a presença do efeito agudo da glicose causando DE. No estudo de Houben (44), o efeito da infusão aguda de glicose por 24 horas em indivíduos normais não apresentou alterações significativas na dilatação vascular na artéria do antebraço induzida por acetilcolina (Ach), nitroprussiato, LNMMA ou norepinefrina, nem na microcirculação da pele. As diferenças entre os estudos se devem a diferenças metodológicas, e pela ação vasodilatadora da insulina, que aumenta compensatoriamente quando se infunde glicose em indivíduos normais. $\mathrm{O}$ aumento da insulina pode mascarar a disfunção aguda do endotélio, proporcionando vasodilatação. Nos estudos em que se bloqueia a ação da insulina com octreotide, $o$ efeito agudo da hiperglicemia isolada fica evidente (43).

\section{DE NO DM1 COM NORMO E MICROALBUMINÚRIAS}

A presença de microalbuminúria no DMl está fortemente associada à DE. No estudo de Dogra e cols. (49), pacientes com 25 anos de DMl, microalbuminúria e controle glicêmico inadequado ( $\mathrm{HbAlc} 8,5 \%$ ) foram comparados a pacientes DMl normoalbuminúricos e a indivíduos não-diabéticos. Em relação aos não-diabéticos, a vasodilatação mediada pelo endotélio estava comprometida, tanto nos microalbuminúricos como nos normoalbuminúricos, mas foi mais grave nos microalbuminúricos em relação aos normoalbuminúricos (Tabela 2). Neste estudo, a albuminúria foi um predictor independente de DE. Assim, em estudo semelhante envolvendo crianças e adolescentes DMl com menos de cinco anos de doença, houve correlação negativa entre dilatação endotélio-dependente e microalbuminúria $(\mathrm{r}=-0,50, \mathrm{p}=0,049)(50)$.

Embora estudos vasculares em pacientes com DMl e microalbuminúria tenham demonstrado associação definida com a DE $(49,50)$, os resultados são menos claros no que diz respeito a pacientes normoalbuminúricos. Em dois estudos observa-se comprometimento 
Tabela 2. Estudos com dilatação mediada por fluxo e espessura íntima-média de carótida em pacientes com DM1.

\begin{tabular}{|c|c|c|c|c|c|c|c|c|c|c|c|}
\hline \multirow[t]{3}{*}{ Estudo } & \multirow[t]{3}{*}{$\mathbf{N}$} & \multirow[t]{3}{*}{ Idade } & \multirow{3}{*}{$\begin{array}{l}\text { Tempo } \\
\text { DM1 }\end{array}$} & \multirow[t]{3}{*}{ MA } & \multirow[t]{3}{*}{ HbAlc } & \multicolumn{4}{|c|}{ Dilatação mediada por fluxo (\%) } & \multicolumn{2}{|c|}{$\operatorname{EIMC}(\mathrm{mm})$} \\
\hline & & & & & & \multicolumn{2}{|c|}{ ED (\%) } & \multicolumn{2}{|c|}{ El (\%) } & \multirow[t]{2}{*}{ DMI } & \multirow[t]{2}{*}{ Controles } \\
\hline & & & & & & DM1 & Controles & DM1 & Controles & & \\
\hline Lekakis e cols. (52) & 31 & 32 & $\begin{array}{l}N: 13 \\
M: 20\end{array}$ & Sim & $\begin{array}{l}N: 6,5 \\
M: 7,1\end{array}$ & $\begin{array}{c}N: 5,8 \pm 7^{*} \\
M: 0,7 \pm 2,5^{*}\end{array}$ & $\begin{array}{c}11,0 \pm 7 \\
-\end{array}$ & $\begin{array}{l}\text { N: } 19,0 \pm 6,9^{*} \\
\text { M: } 15,0 \pm 2,9^{*}\end{array}$ & $\begin{array}{c}24,0 \pm 9,0 \\
-\end{array}$ & - & - \\
\hline Dogra e cols.(49) & 34 & $\begin{array}{l}\text { N } 44 \\
\text { M } 48\end{array}$ & $\begin{array}{l}\text { N } 20 \\
\text { M } 25\end{array}$ & Sim & $8,5-8,7$ & $\begin{array}{l}N: 5,4 \pm 0,6^{*} \\
M: 3,2 \pm 0,3^{*}\end{array}$ & $\begin{array}{c}7,9 \pm 0,6 \\
-\end{array}$ & $\mathrm{M}: 11, \overline{9} \pm 1,1^{*}$ & $\begin{array}{c}20,0 \pm 1,2 \\
-\end{array}$ & - & - \\
\hline Singh e cols. (45) & 31 & 15 & 7 & Não & 8,6 & $4,2 \pm 3,8^{*}$ & $8,2 \pm 4,2$ & $17,0 \pm 6,0$ & $18,0 \pm 6,0$ & $0,33 \pm 0,05$ & $0,32 \pm 0,08$ \\
\hline Meecking e cols. (51) & 18 & 38 & 28 & Sim & 10,5 & $\begin{array}{l}N: 2,4^{*}(1,0-3,8) \\
M 2,3^{*}(0,7-3,9)\end{array}$ & $6,3(5,1-7,5)$ & $\begin{array}{l}N: 14,7(10,7-18,7) \\
M: 5,2(11,2-19,2)\end{array}$ & $18,7(16,1-21,3)$ & & \\
\hline Järvisalo e cols. (10) & 45 & 11 & 4 & Não & 8,9 & $4,4 \pm 3,4^{*}$ & $8,7 \pm 3,3$ & - & - & $0,58 \pm 0,05^{\star}$ & $0,54 \pm 0,04$ \\
\hline Ladeia e cols. (50) & 18 & 13 & 3 & $80 \%$ & 9,3 & $10,9 \pm 2,0$ & $11,2 \pm 2,4$ & - & - & - & - \\
\hline Ceriello e cols. (54) & 36 & 28 & 9 & Não & 8,3 & $5,5 \pm 0,5^{*}$ & $10,8 \pm 0,8$ & & & - & - \\
\hline
\end{tabular}

ED: endotélio-dependente; El: endotélio-independente; N: normoalbuminúricos, Mi: microalbuminúricos, ${ }^{*} \mathrm{P}<0,05$. EIMC: Espessura intima-média da carótida * $\mathrm{P}<0,05$ versus controles, ED: dilatação endotélio-dependente (\% em relação ao basal).

da vasodilatação endotélio-dependente em pacientes DMl e normoalbuminúria. No estudo de Meeking e cols. (51) pacientes com normoalbuminúria e longa duração do diabetes ( 27 anos) e com controle glicêmico insatisfatório (HbAlc média 9,6\%) apresentaram comprometimento da vasodilatação mediada por fluxo em relação aos controles não-diabéticos. Da mesma forma, Lekakis e cols. (52) observaram comprometimento da função endotelial em pacientes DMl normoalbuminúricos com 13 anos de duração, porém mais jovens (32 anos de idade, em média) e com controle glicêmico adequado (HbAlc média 6,5\%), sugerindo que a DE possa ser um evento ainda mais precoce do que a microalbuminúria ao longo da história natural do DMI. Em contraposição, no estudo de Enderle e cols. (53), pacientes com DMl normoalbuminúricos com idade média 41,5 anos, 21 anos de duração do diabetes e controle glicêmico inadequado ( $\mathrm{HbAlc} 8,0 \%$ ) não mostrou diferença na vasodilatação endotélio-dependente em relação aos controles não-diabéticos.

As possíveis explicações para estas diferenças passam por questões metodológicas, especialmente de aferição, em que é possível que ocorram diferenças significativas de sensibilidade do ultra-som de alta resolução (49). Outro fator importante parece ser a variabilidade da glicemia, que pode interferir significativamente na função endotelial (54).

\section{EFEITO DO CONTROLE GLICÊMICO A LONGO PRAZO}

A associação entre HbAlc e dilatação mediada por fluxo (DMF) foi estudada em número relativamente pequeno de pacientes com diabetes e é mal definida. No estudo de Ladeia e cols. (50), em 19 pacientes DMl normo e microalbuminúricos, observou-se correlação positiva entre DMF e HbAlc, $(r=0,53, p=0,002)$. Ao contrário, no estudo de Lekakis e cols. (52), o aumento da HbAlc parece ter impacto na DMF. Neste estudo transversal, pacientes DMl com HbAlc > 6\% apresentaram significativo comprometimento da função endotelial, quando comparados aos pacientes com $\mathrm{HbAlc}<6 \%$.

Em estudo realizado por nosso grupo, avaliando 13 pacientes com DM2 normoalbuminúricos, com bom controle glicêmico (HbAlc média 6,5\%), observou-se uma correlação negativa entre a HbAlc e a DMF $(r=$ $-0,37, p=0,0028$ ). Quando comparados os grupos com $\mathrm{DE}$ e sem DE, a HbAlc foi maior no grupo com DE, $6,84(0,24)$ versus $6,18(0,14), \mathrm{p}=0,004$, respectivamente. Entre os 11 pacientes com melhor controle, sete apresentavam DE (55). A principal explicação para estas discrepâncias deve-se ao fato de que a HbAlc não reflete as variações agudas da glicemia, que ocorrem no diabetes, embora seja um fator de risco para as complicações 
micro e macrovasculares do diabetes (56). A glicemia de jejum, por sua vez, correlaciona-se com a redução percentual da DMF (45).

O efeito da variação glicêmica foi estudado por Sorensen e cols. (57) em pacientes com DMl normoalbuminúricos, em que foi avaliado o impacto da deterioração aguda do controle metabólico na $\mathrm{DE}$. Todos os pacientes foram avaliados com DMF e marcadores sorológicos de função endotelial após 48 horas de bom ou mau controle metabólico, após randomização. Este último induzido após redução da dose de insulina em $20 \%$ a $30 \%$ e liberação da dieta, por período de três semanas. A média da glicemia no período de bom controle foi $113 \mathrm{mg} / \mathrm{dl}$ comparada a $286 \mathrm{mg} / \mathrm{dl}$ no período de mau controle. Ambas vasodilatação endotélio-dependente e endotélio-independente foram significativamente menores no período de piora do controle glicêmico em relação ao período de bom controle glicêmico. Da mesma forma, houve aumento significativo do fator de von Willebrand após a deterioração do controle. Estes resultados indicam que, no DMl, a função endotelial sofre impacto significativo da variação aguda da glicemia, porém pode ser revertida com a melhora do controle glicêmico.

O efeito da glicemia pós-prandial na função endotelial tem sido pouco estudado no DMl. Ceriello e cols. (58) estudaram o impacto da glicemia pós-prandial e da hipertrigliceridemia pós-prandial na função endotelial de pacientes com DM2, e observaram que marcadores sorológicos de $\mathrm{DE}$, como a concentração sérica de moléculas de adesão ICAM-1, E-selecina, VCAM-l e marcadores de estresse oxidativo, como a nitrotirosina, aumentavam agudamente após a ingestão de $75 \mathrm{~g}$ de glicose oral. Este efeito, por sua vez, era ainda maior quando o estudo era realizado adicionalmente com uma sobrecarga lipídica (58), indicando que tanto a hiperglicemia aguda, como a lipemia aguda, interferem marcadamente na função endotelial no diabetes. Em outro estudo, os mesmos autores (59) observaram que após uma sobrecarga oral de glicose, a redução da capacidade de dilatação vascular mediada pelo endotélio acentua-se até a segunda hora, mas retorna ao basal ao completar quatro horas da sobrecarga de glicose. Com a sobrecarga lipídica, entretanto, a DMF permanece alterada até quatro horas após. Estes dados sugerem que o efeito da glicemia pós-prandial é independente do efeito da lipemia pós-prandial e os autores destacam que ambas são mediadas pelo aumento do estresse oxidativo.

\section{EFEITO DO TEMPO DE DM 1 NA FUNÇÃO ENDOTELIAL}

O tempo de diabetes é um determinante importante para a presença de DE no DMl. Em pacientes com mais de 10 anos de doença, a DE é um achado relativamente comum.

Entre cinco e dez anos de DMl, a DE ocorre mais ocasionalmente. No estudo de Singh e cols., 31 adolescentes com pelo menos um ano de DMl (média 6,8 anos) e mau controle glicêmico (HbAlc 8,6\%) comparados a indivíduos sem diabetes apresentaram comprometimento da vasodilatação endotélio-dependente (45). Neste estudo não houve diferença na vasodilatação endotélio-independente nem na espessura da camada íntima-média da carótida (45).

A duração do diabetes apresentou correlação inversa com a dilatação endotélio-dependente $(r=-0,39$, $p=0,02)$. Estes dados sugerem que a DE pode ocorrer na primeira década de DMl e é mais precoce do que o aumento da espessura da camada intima-média das carótidas.

Dados mais recentes indicam que a DE pode ser ainda mais precoce, ocorrendo antes de cinco anos do início da doença, inclusive precedendo a microalbuminúria. Järvisalo e cols. (10) compararam crianças de 11 anos de idade, com DMl há quatro anos, não-obesas, com mau controle do DMl (HbAlc 9,8\%) a crianças sem DM, em relação à DMF e à espessura íntima-média da carótida e evidenciaram a presença de DE em 36\% dos casos (10). Neste estudo, a espessura íntima-média da carótida foi maior nas crianças com DMl. Os autores concluíram que DE é comum em crianças com $\mathrm{DMl}$ inicial e pode ser um predictor para o desenvolvimento de aterosclerose prematura.

Em contrapartida, Ladeia e cols., avaliando adolescentes com 3 anos de DMl, com mau controle glicêmico (HbAlc 9,35\%), não encontraram diferença na DMF entre pacientes e controles (50). Os dados dos estudos referidos anteriormente permitem inferir que a DE começa a surgir entre três e cinco anos após o início do DMl.

\section{MECANISMOS DE DE NO DIABETES TIPO 1}

Os mecanismos pelo qual o diabetes tipo 1 leva à $\mathrm{DE}$ são complexos e parcialmente compreendidos. Embora a presença concomitante de hipertensão e dislipide- 
mia possa contribuir diretamente para a DE, uma combinação de diversos mecanismos diretamente relacionados são determinantes para o desenvolvimento desta condição.

\section{Estresse oxidativo}

Tem sido sugerido que a DE induzida pela hiperglicemia seja mediada por radicais livres derivados do metabolismo do ácido araquidônico (60). Em células endoteliais de aorta humana, a exposição prolongada a altas concentrações de glicose aumenta a expressão gênica do eNOS e a liberação de NO (61). Contudo, ocorre aumento concomitante do radical superóxido, um potente oxidante. Estes ânions inativam o $\mathrm{NO} e$ levam à produção de peroxinitrito (ONOO-), um ativador da peroxidação lipídica e da produção de prostanóides.

Segundo Brownlee (63), a hiperglicemia leva à produção de superóxido nas células endoteliais em nível mitocondrial e está implicada na gênese das complicações do DM. O ânion superóxido liga-se ao NO, prejudicando a sua ação no endotélio (64). Além disso, a produção aumentada de superóxido ativa a proteína quinase $\mathrm{C}$, que por sua vez induz a síntese da enzima NADPH oxidase, que também contribui para produção de superóxido. A hiperglicemia também favorece, por meio da ativação do NFkB, aumento da expressão do eNOS com geração de NO (65). As superproduções de superóxido e de NO favorecem a formação de peroxinitrito, que apresenta ação citotóxica, interferindo no cofator para produção de NO, tetrahidrobiopterina, promovendo também produção de superóxido, em vez de NO (62).

\section{Produtos de glicação avançada}

$\mathrm{Na}$ presença de hiperglicemia sustentada, proteínas teciduais, como o colágeno da parede arterial, sofrem glicação não enzimática e ligações cruzadas, resultando a formação de produtos finais de glicação avançada (AGEs). Estes produtos possivelmente promovem DE por meio de modificações químicas permanentes, estimulando resposta celular pelos receptores específicos (66). Eles têm sido identificados em macrófagos humanos (67), os quais tem um papel importante na eliminação de proteínas glicadas modificadas. O efeito dos AGEs é o de reduzir a disponibilidade do $\mathrm{NO}$, visto que os intermediários reativos na via de formação podem fixar o NO e comprometer o seu efeito antiproliferativo.

\section{Ativação da via dos polióis}

A hiperglicemia crônica aumenta a atividade da aldose redutase e leva à ativação da via dos polióis com transformação da glicose em sorbitol e subseqüentemente em frutose, promovendo consumo de NADPH, cofator importante para síntese de NO (68). Por ser um importante cofator para a NOS na síntese de NO, a sua depleção leva à redução da produção de NO. Permanece em aberto, entretanto, a sua importância na prevenção da aterosclerose humana.

\section{Proteína quinase C}

A ativação da proteína $\mathrm{C}$ quinase (PKC) por meio do aumento do diacil-glicerol (DAG) induzido pela hiperglicemia também tem sido sugerida como mecanismo para DE e complicações vasculares no diabetes. Esta ativação está associada ao aumento da albuminúria em ratos (69). A PKC está associada a diversas alterações vasculares, como uma diminuição da $\mathrm{Na}+/ \mathrm{K}+$ ATPase, ao aumento da matriz extracelular, ao aumento de permeabilidade, de contratilidade e a proliferação celular.

\section{DE COMO MARCADOR DE RISCO CARDIOVASCULAR NO DM 1}

A relação entre $\mathrm{DE}$ e doença cardiovascular é bem conhecida. A DE e a inflamação crônica (low-grade inflammation) estão implicadas na patogênese da doença aterotrombótica cardiovascular em indivíduos com ou sem diabetes, independentemente da presença de fatores de risco, como hipertensão, dislipidemia, tabagismo. O primeiro estudo a demonstrá-la avaliou pacientes com doença coronariana leve, não-obstrutiva e sem diabetes, os quais eram submetidos à avaliação da reatividade vascular por meio da administração intracoronáriana de acetilcolina, adenosina e nitroglicerina, seguido de ultra-som intravascular. Os pacientes eram estudados longitudinalmente para avaliação de desfechos cardiovasculares (70). Neste estudo, os pacientes foram divididos de acordo com a gravidade da DE e acompanhados por 28 meses em média. Ao final do seguimento, $14 \%$ dos pacientes com DE grave apresentaram eventos cardiovasculares $(\mathrm{p}<0,05)$, enquanto nenhum dos pacientes com DE leve ou ausente tiveram desfechos.

$\mathrm{O}$ valor preditivo para mortalidade cardiovascular de marcadores de DE como o fator de von Willebrand (vWf) foi avaliado no estudo HOORN (71). Este estu- 
do foi uma coorte populacional de 2.484 indivíduos caucasianos com 50 a 70 anos de idade, entre 1989 e 1992, em que $27 \%$ apresentavam DM2 e $27 \%$ apresentavam intolerância à glicose (71). Após cinco anos de acompanhamento, 58 indivíduos evoluíram a óbito. Quando se avaliou o vWf comparando os níveis encontrados no tercil superior $(>1,56 \mathrm{UI} / \mathrm{ml})$ em relação aos dois tercis inferiores $(<1,56 \mathrm{UI} / \mathrm{ml})$, o risco de mortalidade cardiovascular em pacientes com diabetes foi de 2,30 (IC95\% 0,80-6,64) após ajustes para idade, sexo, tolerância à glicose, enquanto em pacientes sem diabetes o risco foi de 4,10 (IC 95\%: 0,96-17,54). Em todos os indivíduos agrupados, o risco relativo para mortalidade por todas as causas associado ao vWf no tercil superior foi de 2,03 (IC95\%: 1,19-3,47). Estes resultados sugerem que o vWf, como marcador de DE, seja um preditor independente de morte cardiovascular (71).

Em pacientes com DMl, o vWf está, em geral, aumentado em comparação com indivíduos saudáveis (72). Este aumento é maior na presença de micro e macroalbuminúrias em relação aos pacientes normoalbuminúricos e correlaciona-se positivamente com a proteína $\mathrm{C}$ reativa $(r=0,44, p<0,0005)$, indicando associação entre DE e inflamação vascular (72). Os mecanismos pelos quais o risco cardiovascular está associado a níveis elevados de vWf não são bem conhecidos, mas refletem DE generalizada e um estado pró-trombótico (73), o que representa risco maior de desenvolvimento de doença aterosclerótica (74). Como o vWf também pode ser originado de plaquetas, a medida combinada de vWf e de AP-t pode ser um índice mais específico e sensível de alteração da célula endotelial (75).

Outros marcadores plasmáticos de DE incluem ativador de plasminogênio tecidual (AP-t), E-selectina, molécula de adesão à célula vascular, molécula de adesão intracelular e fator de crescimento endotelial vascu$\operatorname{lar}(58)$.

Marcadores inflamatórios, como o TNF- $\alpha$, IL-6 e proteína $\mathrm{C}$ reativa, estão positivamente associados ao risco de doença vascular em indivíduos sem diabetes (76). Em pacientes com DMl, marcadores inflamatórios, como a proteína $\mathrm{C}$ reativa ultra-sensível (CRP), interleucina-6 (IL-6) e fator de necrose tumoral alfa (TNF- $\alpha$ ), também são importantes determinantes de inflamação. No estudo (EURODIAB Prospective Complications Study (7), um estudo caso-controle aninhado de 348 pacientes obtido de 543 participantes do $E U$ RODIAB IDDM Complications Study Group foram analisados marcadores inflamatórios como CRP,
TNF- $\alpha$, IL-6 em indivíduos com DMl e indivíduos sem diabetes e comparados com os níveis de marcadores de $\mathrm{DE}$, como a E-seletina e a molécula vascular da célula de adesão-1 (VCAM-1). As medidas de inflamação estiveram diretamente associadas com duração do diabetes, controle glicêmico, perfil lipídico, pressão sistólica e com marcadores de DE (7). Já a proteína C reativa apresenta-se como preditor de todas as causas de mortalidade cardiovascular, estando relacionada com outros fatores de risco (71).

\section{MANEJO CLÍNICO DA DE NO DM1}

O manejo da DE no DMl requer bom controle glicêmico, com ênfase, principalmente nas glicemias pósprandiais (59). Níveis adequados de pressão arterial, manejo da dislipidemia, suspensão do tabagismo, dieta pobre em gorduras e estímulo à prática da atividade física também são importantes $(77,78)$. O benefício do

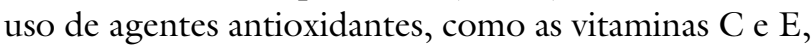
é controverso $(79,80)$. No entanto, a associação da vitamina $\mathrm{C}$ ao bom controle glicêmico foi capaz de melhorar a DE no DM (54).

$\mathrm{O}$ uso da atorvastatina em pacientes com dislipidemia, sem DM e com DE, evidenciou que a dose de 40 $\mathrm{mg}$ /dia melhora a FMD (2,6\% para 4,0\% e 6\% após o uso respectivamente por 1 a 6 semanas) (81). Em indivíduos com DMl e microalbuminúria, o uso da estatina parece apresentar efeito nas FMD endotélios-dependente e independente, provavelmente secundária a sua ação pleiotrófica, reduzindo o estresse oxidativo e aumentando a disponibilidade do NO (82).

$\mathrm{O}$ uso de inibidores da enzima conversora da angiotensina II (IECA em 91 indivíduos com DMl, sem complicações vasculares) não demonstrou benefício na melhora da função endotelial (83). Já pacientes com DMl normotensos, mas com microalbuminúria, os IECA parecem melhorar as vasodilatações endotélios-dependente e independente (84). A combinação do uso de inibidores do receptor da enzima conversora de angiotensina II (irbesartan) com a estatina em indivíduos com DM2 parece melhorar o estresse oxidativo, o processo inflamatório e com isso a DE (85).

\section{CONCLUSÕES}

A DE é um fenômeno precoce na patogênese da aterosclerose no DMl e pode estar presente até mesmo antes 
do surgimento da microalbuminúria. O seu uso clínico, embora ainda não totalmente definido, é promissor como avaliação prognóstica na prevenção primária de eventos cardiovasculares no DMl. Medidas terapêuticas com potencial para reduzir os efeitos da DE no DMl vem sendo agregadas ao arsenal terapêutico do diabetes e incluem o controle intensivo da glicemia, o uso de estatinas e inibidores do sistema renina-angiotensina. Assim, é importante que se definam, em estudos futuros, se a normalização da função endotelial pode ser traduzida em redução de morbimortalidade.

\section{AGRADECIMENTOS}

Suporte Financeiro: Conselho Nacional de Desenvolvimento Científico e Tecnológico ( $\mathrm{CNPq}$ ) e Coordenação de Aperfeiçoamento de Pessoal de Nível Superior (Capes), Brasil.

\section{REFERÊNCIAS}

1. The effect of intensive treatment of diabetes on the development and progression of long-term complications in insulindependent diabetes mellitus. The Diabetes Control and Complications Trial Research Group. N Engl J Med. 1993;329: 977-86.

2. UKPDS 28: a randomized trial of efficacy of early addition of metformin in sulfonylurea-treated type 2 diabetes. U.K. Prospective Diabetes Study Group. Diabetes Care. 1998;21:87-92.

3. Epidemiology of Diabetes Interventions and Complications (EDIC). Design, implementation, and preliminary results of a long-term follow-up of the Diabetes Control and Complications Trial cohort. Diabetes Care. 1999;22:99-111.

4. Krolewski AS, Kosinski EJ, Warram JH, Leland OS, Busick EJ, Asmal AC, et al. Magnitude and determinants of coronary artery disease in juvenile-onset, insulin-dependent diabetes mellitus. Am J Cardiol. 1987;59:750-5.

5. Morrish NJ, Stevens LK, Fuller JH, Keen H, Jarrett RJ. Incidence of macrovascular disease in diabetes mellitus: the London cohort of the WHO Multinational Study of Vascular Disease in Diabetics. Diabetologia. 1991;34:584-9.

6. Kannel WB, McGee DL. Diabetes and cardiovascular disease. The framingham study. JAMA. 1979;241:2035-8.

7. Schram MT, Chaturvedi N, Schalkwijk C, Giorgino F, Ebeling $P_{\text {, }}$ Fuller $\mathrm{JH}$, et al. Vascular risk factors and markers of endothelial function as determinants of inflammatory markers in type 1 diabetes: the EURODIAB Prospective Complications Study. Diabetes Care. 2003;26:2165-73.

8. Stehouwer CD, Gall MA, Twisk JW, Knudsen E, Emeis JJ, Parving $\mathrm{HH}$. Increased urinary albumin excretion, endothelial dysfunction, and chronic low-grade inflammation in type 2 diabetes: progressive, interrelated, and independently associated with risk of death. Diabetes. 2002;51:1157-65.

9. Nathan DM, Cleary PA, Backlund JY, Genuth SM, Lachin JM, Orchard TJ, et al. Intensive diabetes treatment and cardiovas- cular disease in patients with type 1 diabetes. N Engl J Med. 2005;353:2643-53.

10. Järvisalo MJ, Raitakari M, Toikka JO, Putto-Laurila A, Rontu R, Laine $S$, et al. Endothelial dysfunction and increased arterial intima-media thickness in children with type 1 diabetes. Circulation. 2004;109:1750-5.

11. Juonala M, Viikari JS, Laitinen T, Marniemi J, Helenius H, Ronnemaa $T$, et al. Interrelations between brachial endothelial function and carotid intima-media thickness in young adults: the cardiovascular risk in young finns study. Circulation. 2004;110:2918-23.

12. Palmer RM, Ferrige AG, Moncada S. Nitric oxide release accounts for the biological activity of endothelium-derived relaxing factor. Nature. 1987;327:524-6.

13. Moncada S, Higgs A. The L-arginine-nitric oxide pathway. N Engl J Med. 1993;329:2002-12.

14. Pohl U, Busse R. Hypoxia stimulates release of endotheliumderived relaxant factor. Am J Physiol. 1989;256:H1595-1600.

15. Hutcheson IR, Griffith TM. Release of endothelium-derived relaxing factor is modulated both by frequency and amplitude of pulsatile flow. Am J Physiol. 1991;261:H257-262.

16. Vascular endothelial cells synthesize nitric oxide from L-arginine. Nature. 1988;333:664-6.

17. Vallance P, Collier J, Moncada S. Effects of endothelium-derived nitric oxide on peripheral arteriolar tone in man. Lancet. 1989;2:997-1000.

18. Nathan DM, Lachin J, Cleary P, Orchard T, Brillon DJ, Backlund $J Y$, et al. Intensive diabetes therapy and carotid intima-media thickness in type 1 diabetes mellitus. $\mathrm{N}$ Engl $\mathrm{J}$ Med. 2003;348:2294-303.

19. Pomilio M, Mohn A, Verrotti A, Chiarelli F. Endothelial dysfunction in children with type 1 diabetes mellitus. J Pediatr Endocrinol Metab. 2002;15:343-61.

20. Vita JA, Keaney Jr. JF, Endothelial function: a barometer for cardiovascular risk? Circulation. 2002;106:640-2.

21. Kato M, Shiode N, Yamagata T, Matsuura H, Kajiyama G. Coronary segmental responses to acetylcholine and bradykinin in patients with atherosclerotic risk factors. Am J Cardiol. 1997;80:751-5.

22. Benjamin N, Calver A, Collier J, Robinson B, Vallance P, Webb $D$. Measuring forearm blood flow and interpreting the responses to drugs and mediators. Hypertension. 1995;25:918-23.

23. Khan F, Elhadd TA, Greene SA, Belch JJ. Impaired skin microvascular function in children, adolescents, and young adults with type 1 diabetes. Diabetes Care. 2000;23:215-20.

24. Joannides R, Haefeli WE, Linder L, Richard V, Bakkali EH, Thuillez $\mathrm{C}$, et al. Nitric oxide is responsible for flow-dependent dilatation of human peripheral conduit arteries in vivo. Circulation. 1995;91:1314-9.

25. Corretti MC, Anderson TJ, Benjamin EJ, Celermajer D, Charbonneau $F$, Creager MA, et al. Guidelines for the ultrasound assessment of endothelial-dependent flow-mediated vasodilation of the brachial artery: a report of the International Brachial Artery Reactivity Task Force. J Am Coll Cardiol. 2002;39:257-65.

26. Pedro MA CS, Colombo FMC. Métodos de investigação do endotélio. In: Luz PL, Laurindo FRM, Chagas ACP. Endotélio. Doenças cardiovasculares. São Paulo: Atheneu, 2003.

27. Mahmud FH EM, Lee RA, Lteif AN, Driscoll DJ, Lerman A. Altered endothelial function in asymptomatic male adolescents with type 1 diabetes. Congenital Heart Disease. 2006;1:98-103. 
28. Haller MJ, Stein J, Shuster J, Theriaque D, Silverstein J, Schatz DA, et al. Peripheral artery tonometry demonstrates altered endothelial function in children with type 1 diabetes. Pediatr Diabetes. 2007;8:193-8.

29. Aellig WH. A new technique for recording compliance of human hand veins. Br J Clin Pharmacol. 1981;11:237-43.

30. Signori LU, Plentz RDM, Moreno Jr H, Gelonese B, Irigoyen MC. Reduced venous endothelial responsiveness after oral lipid overload in healthy volunteers. Metabolism. 2007 [in press].

31. Higashi Y, Sasaki S, Nakagawa K, Matsuura H, Kajiyama G, Oshima T. A noninvasive measurement of reactive hyperemia that can be used to assess resistance artery endothelial function in humans. Am J Cardiol. 2001;87:121-125, A129.

32. Hadcock $S$, Richardson M, Winocour PD, Hatton MW. Intimal alterations in rabbit aortas during the first 6 months of alloxan-induced diabetes. Arterioscler Thromb. 1991;11:517-29.

33. Lin SJ, Hong CY, Chang MS, Chiang BN, Chien S. Increased aortic endothelial death and enhanced transendothelial macromolecular transport in streptozotocin-diabetic rats. Diabetologia. 1993;36:926-30.

34. Aageneas $\mathrm{O} \mathrm{MH}$. Light and electron microscopy study of skin capillaries of diabetics. Diabetes. 1961;10:253-9.

35. Rosenstock J, Challis P, Strowig S, Raskin P. Improved diabetes control reduces skeletal muscle capillary basement membrane width in insulin-dependent diabetes mellitus. Diabetes Res Clin Pract. 1988;4:167-75.

36. Cester N, Rabini RA, Salvolini E, Staffolani R, Curatola A, Pugnaloni $A$, et al. Activation of endothelial cells during insulindependent diabetes mellitus: a biochemical and morphological study. Eur J Clin Invest. 1996;26:569-73.

37. Oyama $Y$, Kawasaki H, Hattori $Y$, Kanno M. Attenuation of endothelium-dependent relaxation in aorta from diabetic rats. Eur J Pharmacol. 1986;132:75-8.

38. Tesfamariam B, Jakubowski JA, Cohen RA. Contraction of diabetic rabbit aorta caused by endothelium-derived PGH2-TxA2. Am J Physiol. 1989;257:H1327-33.

39. Calver A, Collier J, Vallance P. Inhibition and stimulation of nitric oxide synthesis in the human forearm arterial bed of patients with insulin-dependent diabetes. J Clin Invest. 1992;90:2548-54.

40. Johnstone MT, Creager SJ, Scales KM, Cusco JA, Lee BK, Creager MA. Impaired endothelium-dependent vasodilation in patients with insulin-dependent diabetes mellitus. Circulation. 1993;88:2510-6.

41. Williams SB, Cusco JA, Roddy MA, Johnstone MT, Creager MA. Impaired nitric oxide-mediated vasodilation in patients with non-insulin-dependent diabetes mellitus. J Am Coll Cardiol. 1996;27:567-74.

42. McVeigh GE, Brennan GM, Johnston GD, McDermott BJ, McGrath LT, Henry WR, et al. Impaired endothelium-dependent and independent vasodilation in patients with type 2 (non-insulin-dependent) diabetes mellitus. Diabetologia. 1992;35:771-6.

43. Williams SB, Goldfine AB, Timimi FK, Ting HH, Roddy MA, Simonson DC, et al. Circulation. 1998;97:1695-701.

44. Houben AJ, Schaper NC, de Haan $\mathrm{CH}$, Huvers FC, Slaaf DW, de Leeuw PW, et al. Local 24-h hyperglycemia does not affect endothelium-dependent or -independent vasoreactivity in humans. Am J Physiol. 1996;270:H2014-20.

45. Singh TP, Groehn H, Kazmers A. Vascular function and carotid intimal-medial thickness in children with insulin-dependent diabetes mellitus. J Am Coll Cardiol. 2003;41:661-5.
46. Steinberg HO, Brechtel G, Johnson A, Fineberg N, Baron AD. Insulin-mediated skeletal muscle vasodilation is nitric oxide dependent. A novel action of insulin to increase nitric oxide release. J Clin Invest. 1994;94:1172-9.

47. Makimattila S, Mantysaari M, Groop PH, Summanen P, Virkamaki A, Schlenzka A, et al. Hyperreactivity to nitrovasodilators in forearm vasculature is related to autonomic dysfunction in insulindependent diabetes mellitus. Circulation. 1997;95:618-25.

48. Elliott TG, Cockcroft JR, Groop PH, Viberti GC, Ritter JM. Inhibition of nitric oxide synthesis in forearm vasculature of insulindependent diabetic patients: blunted vasoconstriction in patients with microalbuminuria. Clin Sci (Lond). 1993;85:687-93.

49. Dogra G, Rich L, Stanton K, Watts GF. Endothelium-dependent and independent vasodilation studies at normoglycaemia in type I diabetes mellitus with and without microalbuminuria. Diabetologia. 2001;44:593-601.

50. Ladeia AM, Ladeia-Frota C, Pinho L, Stefanelli E, Adan L. Endothelial dysfunction is correlated with microalbuminuria in children with short-duration type 1 diabetes. Diabetes Care. 2005;28:2048-50.

51. Meeking DR, Cummings MH, Thorne S, Donald A, Clarkson $P$, Crook JR, et al. Endothelial dysfunction in type 2 diabetic subjects with and without microalbuminuria. Diabet Med. 1999; 16:841-7.

52. Lekakis J, Papamichael C, Anastasiou H, Alevizaki M, Desses $\mathrm{N}$, Souvatzoglou A, et al. Endothelial dysfunction of conduit arteries in insulin-dependent diabetes mellitus without microalbuminuria. Cardiovasc Res. 1997;34:164-8.

53. Enderle MD, Balletshofer BM, Schmulling RM, Haring HU, Pfohl M. [Early detection of atherosclerosis with high resolution ultrasound exemplified by type II diabetic patients]. Ultraschall Med. 1998;19:16-21.

54. Ceriello A, Kumar S, Piconi L, Esposito K, Giugliano D. Simultaneous control of hyperglycemia and oxidative stress normalizes endothelial function in type 1 diabetes. Diabetes Care. 2007;30:649-54.

55. Silva AMV, Bertoluci MC, Rabelo E, Signori LU, Irigoyen MC, Schaan BD. Associação entre a hemoglobina glicada e disfunção endotelial em pacientes com diabetes tipo 2 e bom controle glicêmico. Arq Bras Endocrinol Metab. 2006;50.

56. Bonora E, Calcaterra F, Lombardi S, Bonfante N, Formentini G, Bonadonna RC, et al. Plasma glucose levels throughout the day and $\mathrm{HbA}(1 \mathrm{c})$ interrelationships in type 2 diabetes: implications for treatment and monitoring of metabolic control. Diabetes Care. 2001;24:2023-29.

57. Sorensen VR, Mathiesen ER, Clausen P, Flyvbjerg A, FeldtRasmussen B. Impaired vascular function during short-term poor glycaemic control in type 1 diabetic patients. Diabet Med. 2005;22:871-6.

58. Ceriello A, Quagliaro L, Piconi L, Assaloni R, Da Ros R, Maier A, et al. Effect of postprandial hypertriglyceridemia and hyperglycemia on circulating adhesion molecules and oxidative stress generation and the possible role of simvastatin treatment. Diabetes. 2004;53:701-10.

59. Ceriello A, Taboga C, Tonutti L, Quagliaro L, Piconi L, Bais B, et al. Evidence for an independent and cumulative effect of postprandial hypertriglyceridemia and hyperglycemia on endothelial dysfunction and oxidative stress generation: effects of short- and long-term simvastatin treatment. Circulation. 2002;106:1211-8.

60. Giugliano D, Ceriello A, Paolisso G. Oxidative stress and diabetic vascular complications. Diabetes Care. 1996;19:257-67. 
61. Cosentino F, Hishikawa K, Katusic ZS, Luscher TF. High glucose increases nitric oxide synthase expression and superoxide anion generation in human aortic endothelial cells. Circulation. 1997;96:25-8.

62. Beckman JS, Koppenol WH. Nitric oxide, superoxide, and peroxynitrite: the good, the bad, and ugly. Am J Physiol. 1996;271:C1424-1437.

63. Brownlee M. Biochemistry and molecular cell biology of diabetic complications. Nature. 2001;414:813-20.

64. Nishikawa T, Edelstein D, Du XL, Yamagishi S, Matsumura T, Kaneda $Y$, et al. Normalizing mitochondrial superoxide production blocks three pathways of hyperglycaemic damage. Nature. 2000;404:787-90.

65. Spitaler MM, Graier WF. Vascular targets of redox signalling in diabetes mellitus. Diabetologia. 2002;45:476-94.

66. Brownlee M, Cerami A, Vlassara H. Advanced glycosylation end products in tissue and the biochemical basis of diabetic complications. N Engl J Med. 1988;318:1315-21.

67. Vlassara H, Brownlee M, Cerami A. High-affinity-receptor-mediated uptake and degradation of glucose-modified proteins: a potential mechanism for the removal of senescent macromolecules. Proc Natl Acad Sci USA. 1985;82:5588-92.

68. Shkhvatsabaya IK, Erina EV, Almusaui Al. Venous tone in essential hypertension. Cor Vasa. 1977;19:184-96.

69. Wolf BA, Williamson JR, Easom RA, Chang K, Sherman WR, Turk J. Diacylglycerol accumulation and microvascular abnormalities induced by elevated glucose levels. J Clin Invest. 1991;87:31-8.

70. Suwaidi JA, Hamasaki S, Higano ST, Nishimura RA, Holmes DR, Jr., Lerman A. Long-term follow-up of patients with mild coronary artery disease and endothelial dysfunction. Circulation. 2000;101:948-54.

71. Jager A, van Hinsbergh VW, Kostense PJ, Emeis JJ, Yudkin JS, Nijpels G, et al. von Willebrand factor, C-reactive protein, and 5-year mortality in diabetic and nondiabetic subjects: the Hoorn Study. Arterioscler Thromb Vasc. 1999;Biol 19:3071-8.

72. Schalkwijk CG, Poland DC, van Dijk W, Kok A, Emeis JJ, Drager AM, et al. Plasma concentration of C-reactive protein is increased in type I diabetic patients without clinical macroangiopathy and correlates with markers of endothelial dysfunction: evidence for chronic inflammation. Diabetologia. 1999;42: 351-7.

73. Mannucci PM. von Willebrand factor: a marker of endothelial damage? Arterioscler Thromb Vasc Biol. 1998;18:1359-62.

74. Thompson SG, Kienast J, Pyke SD, Haverkate F, van de Loo JC. Hemostatic factors and the risk of myocardial infarction or sudden death in patients with angina pectoris. European Concerted Action on Thrombosis and Disabilities Angina Pectoris Study Group. N Engl J Med. 1995;332:635-41.

75. Romano M, Pomilio M, Vigneri S, Falco A, Chiesa PL, Chiarelli $F$, et al. Endothelial perturbation in children and adolescents with type 1 diabetes: association with markers of the inflammatory reaction. Diabetes Care. 2001;24:1674-8.
76. Koenig W, Sund M, Frohlich M, Fischer HG, Lowel H, Doring A, et al. C-reactive protein, a sensitive marker of inflammation, predicts future risk of coronary heart disease in initially healthy middle-aged men: results from the MONICA (Monitoring Trends and Determinants in Cardiovascular Disease) Augsburg Cohort Study, 1984 to 1992. Circulation. 1999;99:237-42.

77. Schwab KO, Doerfer J, Krebs A, Krebs K, Schorb E, Hallermann $\mathrm{K}$, et al. Early atherosclerosis in childhood type 1 diabetes: role of raised systolic blood pressure in the absence of dyslipidaemia. Eur J Pediatr. 2007;166:541-8.

78. Roberts CK, Won D, Pruthi S, Lin SS, Barnard RJ. Effect of a diet and exercise intervention on oxidative stress, inflammation and monocyte adhesion in diabetic men. Diabetes Res Clin Pract. 2006;73:249-59.

79. Economides PA, Khaodhiar L, Caselli A, Caballero AE, Keenan $H$, Bursell SE, et al. The effect of vitamin $E$ on endothelial function of micro- and macrocirculation and left ventricular function in type 1 and type 2 diabetic patients. Diabetes. 2005;54:204-11.

80. Beckman JA, Goldfine AB, Gordon MB, Garrett LA, Keaney JF, Jr., Creager MA. Oral antioxidant therapy improves endothelial function in type 1 but not type 2 diabetes mellitus. Am J Physiol Heart Circ Physiol. 2003;285:H2392-8.

81. Taneva E, Borucki K, Wiens L, Makarova R, Schmidt-Lucke C, Luley $\mathrm{C}$, et al. Early effects on endothelial function of atorvastatin $40 \mathrm{mg}$ twice daily and its withdrawal. Am J Cardiol. 2006;97:1002-6.

82. Dogra GK, Watts GF, Chan DC, Stanton K. Statin therapy improves brachial artery vasodilator function in patients with type 1 diabetes and microalbuminuria. Diabet Med. 2005; 22:239-42.

83. Mullen MJ, Clarkson $P$, Donald AE, Thomson $H$, Thorne SA, Powe AJ, et al. Effect of enalapril on endothelial function in young insulin-dependent diabetic patients: a randomized, double-blind study. J Am Coll Cardiol. 1998;31:1330-5.

84. Arcaro G, Zenere BM, Saggiani F, Zenti MG, Monauni T, Lechi $A$, et al. ACE inhibitors improve endothelial function in type 1 diabetic patients with normal arterial pressure and microalbuminuria. Diabetes Care. 1999;22:1536-42.

85. Ceriello A, Assaloni R, Da Ros R, Maier A, Piconi L, Quagliaro $L$, et al. Effect of atorvastatin and irbesartan, alone and in combination, on postprandial endothelial dysfunction, oxidative stress, and inflammation in type 2 diabetic patients. Circulation. 2005;111:2518-24.

\section{Endereço para correspondência:}

Marcello Casaccia Bertoluci

Rua lldefonso Simões Lopes, 99 -

91330-180 - Porto Alegre, RS

E-mail: mbertoluci@uol.com.br 